\title{
Biología del pejerrey Odontesthes bonariensis (Pisces, Atherinopsidae) de la laguna Los Charos (Córdoba, Argentina)
}

\section{Biology of silverside Odontesthes bonariensis (Pisces, Atherinopsidae) in the shallow lake "Los Charos" (Córdoba, Argentina)}

\author{
Miguel Mancini ${ }^{1}$, Ignacio Nicola ${ }^{1}$, Víctor Salinas ${ }^{1}$ y César Bucco ${ }^{2}$
}

\begin{abstract}
1. Acuicultura. Facultad de Agronomía y Veterinaria. Universidad Nacia y Veteinaria. Universidad $36 \mathrm{~km}$ 601. CP 5800. Argentina. Email: mmancini@ayv.unrc.edu.ar 2. Guardafauna ad honorem de la provincia de Córdoba, Argentina.
\end{abstract}

Presentado: 02/06/2008 Aceptado: 07/10/2008 Publicado online: 26/02/2009

\section{Resumen}

El pejerrey Odontesthes bonariensis es la especie más importante de las pesquerías del centro de Argentina. Se estudio la captura por unidad de esfuerzo (CPUE), la condición corporal, el crecimiento y la alimentación de O. bonariensis de la laguna pampeana Los Charos $\left(34^{\circ} 28^{\prime} \mathrm{S}, 64^{\circ} 23^{\prime} \mathrm{W}, 240\right.$ ha), ubicada en la provincia de Córdoba. Se realizaron cuatro muestreos estacionales en el periodo 2002-2003. Para la captura de peces se utilizaron redes de arrastre y enmalle. Se capturaron 2862 ejemplares de un rango de talla comprendido entre 38 y $380 \mathrm{~mm}$ de longitud estándar (LSt). La CPUE promedio fue de 74,3( $\pm 71,0) \mathrm{kg} / 20 \mathrm{hs}$ de tendido de red. La relación LSt-peso presentó diferencias significativas entre épocas del año $(P<0,01)$. Los índices de condición corporal estuvieron dentro de los límites de referencia de la especie. El crecimiento calculado fue: $\mathrm{LSt}_{(t)}=459,8^{*}\left[1-\exp ^{\left(-0,3105^{*}(-0,175)\right)}\right]$. La relación LSt-Longitud total $(\mathrm{LT})$ fue: $\mathrm{LT}(\mathrm{mm})=8,23+\mathrm{LSt}^{*} 1,14(\mathrm{n}=283$; $\left.\mathrm{R}^{2}=0,99\right)$. El zooplancton constituyó un ítem alimenticio secundario en los peces jóvenes. En los ejemplares de 3+ años de vida se observó un marcado canibalismo, situación que explicaría en parte su mejor condición corporal. La laguna Los Charos presenta una elevada producción de O. bonariensis.

Palabras claves: pejerrey, Odontesthes bonariensis, condición, biología, lagunas.

\section{Abstract}

The silverside Odontesthes bonariensis is the most important species in the fisheries of central Argentina. Capture per unit effort (CPUE), body condition, growth and feeding were studied for $O$. bonariensis in the Pampean

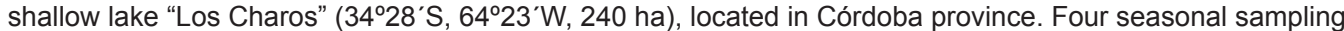
procedures were carried out during 2002-2003. Floating gillnets and trawlnets were used to capture the fishes. Specimens captured $(n=2862)$ were ranged between 38 and $380 \mathrm{~mm}$ of standar length $(S t L)$. CPUE average was $74.3( \pm 71,0) \mathrm{kg} / 20 \mathrm{hs} / \mathrm{gill}$ ets. The StL-weight relationship showed signifficant differences along the seasons $(P<0,01)$. The body score indices were within the reffered limits for this species. Growth was calculated by $\mathrm{StL}_{(t)}=459,8^{*}\left[1-\exp \left(-0,3105^{*}(\mathrm{t}-0,175)\right)\right]$. The StL-Total length $(T L)$ ratio was $T L(\mathrm{~mm})=8,23+\mathrm{StL}^{*} 1,14\left(\mathrm{n}=283 ; \mathrm{R}^{2}=\right.$ 0,99 ). For the young fishes the zooplankton constituted a secundary feeding source. In individuals older than 3 years of age, a marked canibalism was observed, which might explain partially their body condition. The shallow lake "Los Charos" shows an elevated production of $O$. bonariensis.

Keywords: silverside, Odontesthes bonariensis, condition, biology, shallow lakes.

\section{Introducción}

Las lagunas de la región de las Pampas -lagunas pampeanas- de Argentina, son cuerpos de agua someros, en su gran mayoría de formas geométricas sencillas, salinidad muy variable y naturalmente eutróficos (Dangvas 1998; Quirós et al. 2002). Estos lagos de llanura son muy poco profundos y altamente dependientes de las precipitaciones. Quirós (2004), señala para 39 lagunas una profundidad media de $2,1( \pm 1,3) \mathrm{m}$, siendo esta cercana al $70 \%$ de su profundidad máxima.

El pejerrey Odontesthes bonariensis (Valenciennes 1835) es la especie más importante de las pesquerías recreativas, deportivas y comerciales de los ambientes lagunares (López et al. 2001). En la década del 90, numerosas lagunas se incorporaron en forma masiva al circuito productivo y pesquero de esta especie, como respuesta al incremento que generó la demanda, posibilitando la diversificación de los ingresos rurales y un extraordinario movimiento económico (Grosman y Mancini 2001).

Los estudios referidos a la biología pesquera utilizan de manera frecuente diferentes índices de condición corporal. El uso de estos índices radica en su grado de sencillez y fácil interpretación, a la vez que permiten efectuar análisis temporales y espaciales de diferentes poblaciones. Los índices más empleados en $O$. bonariensis son la relación longitud-peso, el índice cefálico y el factor k o índice de condición (Freyre 1976; López et al. 2001). La utilización de algunos índices presenta particularidades debido a la introducción de posibles sesgos, sin embargo condiciones desfavorables del entorno pueden modificar los mismos por lo que resultan de utilidad en muestreos de rutina si se comparan por intervalo de tallas (Freyre et al. 1983). La determinación del crecimiento de $O$. bonariensis es otra herramienta indispensable para una correcta gestión de las pesquerías y presenta íntima asociación con diferentes factores ecológicos y la explotación pesquera de cada ambiente (Baigún y Delfino 2001).

En otro orden, el estudio de la alimentación de los peces permite conocer la ecología de una especie y su lugar en la trama trófica (Bemvenuti 2006). Las características productivas de las lagunas pampeanas favorecen el desarrollo de zooplancton, principal alimento del pejerrey en los primeros ańos de vida (Ringuelet et al. 1980; Escalante 2001)

Si bien se desprende que el estudio del pejerrey ha sido abordado en múltiples ocasiones y bajo diferentes aspectos de su biología, existen en la actualidad cientos de pesquerías donde las poblaciones no han sido evaluadas. Se estudio en este trabajo la abundancia, condición corporal, alimentación y crecimiento de $O$. bonariensis de una laguna pampeana del centro de Argentina.

\section{Materiales y métodos}

Zona de estudio. La laguna Los Charos ( $\left.34^{\circ} 28^{\prime} \mathrm{S}, 64^{\circ} 23^{\prime} \mathrm{W}\right)$, está ubicada en el sur de la provincia de Córdoba, Argentina (Fig. 1). Se encuentra dentro de la llanura pampeana, en una gran unidad ambiental de llanura medanosa (subunidad de de- 

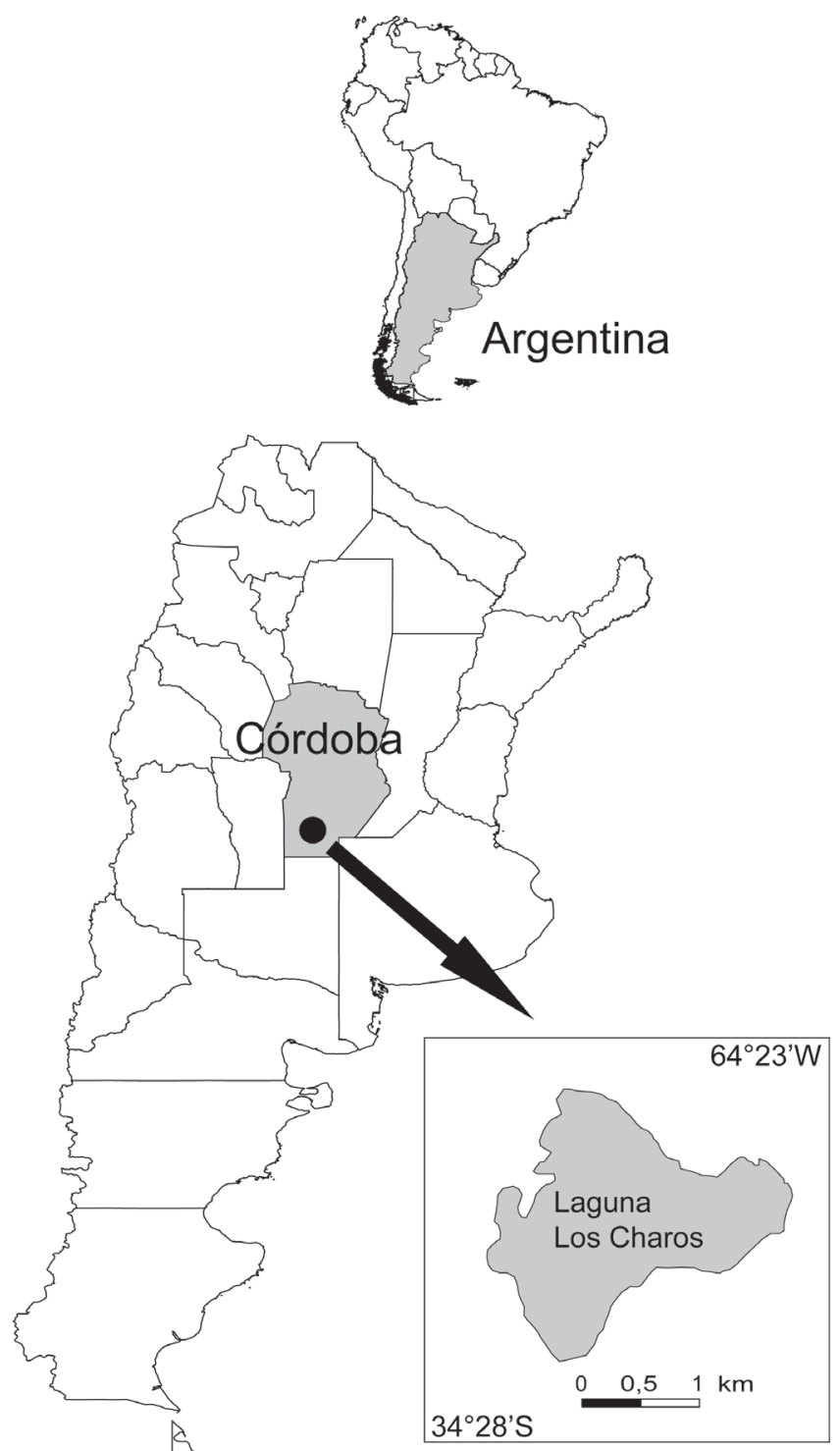

Figura 1. Ubicación geográfica de la laguna Los Charos.

rrames antiguos del río Quinto), en conjunto con un centenar de lagunas permanentes, muchas de las cuales carecen de conexión superficial, por lo que se consideran sistemas cerrados (Cantero et al. 1998). Los Charos posee una superficie de 240 ha y una profundidad media y máxima de 1,78 y $2,47 \mathrm{~m}$ respectivamente. Presenta un elevado estado trófico, agua de característica mesohalina (promedio anual de 9,4 g/L), clorurada sódica y muy dura (Nicola et al. 2007). El ambiente ha sido explotado como pesquería recreativa.

Captura y análisis de la ictiofauna. Para la captura de los peces se realizaron 4 muestreos estacionales durante el periodo 2002-2003 coincidente con los meses de septiembre, diciembre, marzo y mayo para invierno, primavera, verano y otońo respectivamente. Se utilizaron artes de pesca activos y pasivos:

a) Una red de arrastre litoral de $20 \mathrm{~m}$ de largo y copo de 3,5 m.

b) Dos baterías de redes de enmalle flotantes para pesca experimental, compuestas por pańos de 15, 19, 22, 25, 30, 33, 38 y $40 \mathrm{~mm}$ de medida entre nudos (largo total 68,75 m). Las mismas se calaron al atardecer, de manera paralela y perpendicular en relación a la línea de la costa. La captura por unidad de esfuerzo del enmalle (CPUE) se expresó en número y biomasa de peces, con un tiempo de referencia de calado de 20 h. La CPUE promedio se comparó con el registro de $17,3 \mathrm{~kg} / 20 \mathrm{hs}$, correspondiente a la media de las capturas de 23 lagunas pampeanas analizadas con idéntica metodología (Grosman et al. 2001; 2005; Mancini y Grosman 2001; 2004). Además se estimó la biomasa de pejerrey proveniente de la pesca recreativa mediante la cesta media del pescador (García de Jalón Lastra et al. 1993). La misma se expresó en $\mathrm{kg} / \mathrm{ha} / \mathrm{año}$.

En cada muestreo se tomó un mínimo de 60 ejemplares de diferentes intervalos de talla, a los cuales se les tomaron los siguientes caracteres biométricos: longitud de la cabeza (LC), longitud total (LT) y estándar (LSt), todas con una precisión de $1 \mathrm{~mm}$. Para tal fin, se utilizó un ictiómetro y un calibre. Luego se pesó cada ejemplar (peso húmedo, W) mediante una balanza digital (precisión $0,1 \mathrm{~g}$ ) y se extrajeron escamas de la zona típica para estimar el crecimiento.

Por su parte, se determinó el grado de maduración gonadal a través de los 7 estadios descriptos por Calvo y Dadone (1972), considerando como grado 5 al periodo de freza o desove. Además se obtuvieron los siguientes índices de condición, cefálico IC = $\mathrm{LC}^{*} 100 / \mathrm{LSt}$, factor de condición $\mathrm{K}=\mathrm{W}^{*} 10^{5} / \mathrm{LSt}^{3}$, peso relativo $\mathrm{W} r=\mathrm{W} / 4,886 \mathrm{E}^{-6 *} \mathrm{LSt}^{3,179}$, relación entre la longitud y el peso, $\mathrm{W}=a \mathrm{LSt}^{b}$ y la relación longitud total- estándar, $\mathrm{LT}=a+\mathrm{LSt} b$, de acuerdo a lo expresado por Freyre et al. (1976), Royce (1996) y Colautti et al. (2003). Los valores de K y del IC se contrastaron con valores estándar y sus desvíos correspondientes descriptos para la especie por Freyre (1976). Debido a que algunos índices presentan sesgos dados por la talla de los peces (Freyre et al. 1983), los mismos se agruparon por intervalos de LSt.

Para obtener los parámetros de crecimiento se aplicó el método del retrocálculo, cuyos valores fueron ajustados al modelo de Von Bertalanffy, $\mathrm{LSt}_{\mathrm{t}}=\mathrm{L} \infty^{*}\left(1-\mathrm{e}^{-\mathrm{k}^{*}(\mathrm{t}-\mathrm{to})}\right)$ donde $\mathrm{L} \infty$ es la longitud asintótica, $\mathrm{k}$ es la tasa de crecimiento $\mathrm{y}_{0}$ es el tiempo teórico en el cual la longitud de los peces es nula (Moreau 1987). Los resultados fueron cotejados mediante la asignación de edades tentativas a las diferentes modas obtenidas en la distribución de las frecuencias de captura (Sendra y Colautti 1997). Con estos datos se aplicó el índice de performance de crecimiento: $\mathrm{W}=\mathrm{k}$ Lo (Gallucci y Quinn 1979).

Los tractos digestivos fueron colocados en cápsulas de Petri y observados bajo lupa binocular y/o microscopio óptico. Se determinaron los diferentes componentes de la dieta a nivel de grandes grupos biológicos. Se estableció la siguiente escala de abundancia relativa (A): muy abundante, abundante, común, escasa, muy escasa y ausente, cuantificando de 5 a 0 respectivamente. Se calculó la frecuencia absoluta de aparición de cada ítem alimenticio (F) y la diversidad de la dieta mediante el índice de Shannon-Wiener $(\mathrm{H})$. Con estas variables se obtuvo el índice de categorización de ítems $(\mathrm{ICI})=(\mathrm{FxA})^{0,5} / \mathrm{H}$, el cual diferencia componentes primarios ( $\mathrm{ICI}>10)$, secundarios $(5>\mathrm{ICI}>10)$, terciarios $(1>\mathrm{ICI}>5)$ y ocasionales $(\mathrm{ICI}<1)$ de la dieta. Este método es corrientemente utilizado en pejerrey al permitir asignar jerarquías relativas a cada ítem alimenticio (Grosman, 1995).

Los nemátodos encontrados en la cavidad corporal de los peces fueron fijados en alcohol 70\% y glicerina (9:1), aclarados con lactofenol de Amann e identificados mediante claves específicas. 
Tabla 1. Captura por unidad de esfuerzo (CPUE), peso relativo (Wr) y relación LSt-peso de Odontesthes bonariensis en la laguna Los Charos.

\begin{tabular}{lcccc}
\hline & Invierno & Primavera & Verano & Otoño \\
\hline CPUE/20hs ( kg/red) & 181,1 & 59,4 & 34,5 & 19,6 \\
CPUE/20hs (peces/red) & 818,1 & 370,4 & 504,2 & 343,7 \\
Peso medio pez (g) & 221,3 & 160,2 & 68,4 & 57,1 \\
Rango analizado LSt & $43-380$ & $38-378$ & $112-333$ & $48-251$ \\
Wr medio & 94,0 & 89,2 & 86,9 & 90,5 \\
(pendiente regresión) & 3,33 & 3,10 & 2,88 & 3,06 \\
IC $b$ (95\%) & $3,27-3,38$ & $3,04-3,15$ & $2,78-2,95$ & $2,96-3,16$ \\
$a$ (ordenada al origen) & $2,02^{*} 10^{-6}$ & $6,70^{*} 10^{-6}$ & $2,04^{*} 10^{-5}$ & $7,94^{*} 10^{-6}$ \\
$\mathrm{R}^{2}$ ajustado & 0,99 & 0,99 & 0,98 & 0,98 \\
Peces analizados & 73 & 74 & 60 & 71 \\
\hline
\end{tabular}

Se entendió por prevalencia al número de huéspedes positivos divididos por el número de huéspedes totales analizados; por abundancia al promedio de parásitos para el total de peces y por intensidad media al número de parásitos promedio presente en los ejemplares parasitados (Margolis et al. 1982).

Abundancia de zooplancton. De manera conjunta con los muestreos de peces, en 5 sitios de la laguna se filtraron 25 $\mathrm{L}$ de agua por una red $50 \mu \mathrm{m}$ para cuantificar los organismos del zooplancton. Se fijó el material con formalina al $4 \%$ para su posterior lectura mediante cámara abierta tipo Bogorov; la abundancia se calculó de acuerdo a Fabián (1999). Los valores se expresaron en número de organismos/100 L de agua.

Análisis estadístico. La relación $\mathrm{W}=a \mathrm{LSt}^{b}$ se obtuvo por análisis de regresión lineal simple (Weatherley, 1976), donde $a$ es la ordenada al origen y $b$ la pendiente de la regresión calculadas previa transformación logarítmica $\left(\log _{10}\right)$ de los datos: $\log \mathrm{W}=$ $\log a+b^{*} \log L$ St. La existencia de diferencias estadísticas entre las diferentes épocas del año, fue analizada mediante comparación de los intervalos de confianza de los coeficientes y de un análisis de covarianza (ANCOVA), previa verificación del supuesto de homogeneidad de las pendientes (García-Berthou, 2001). La existencia de diferencias significativas de abundancia de zooplancton y $\mathrm{Wr}$ entre muestreos se realizó mediante la prueba de Kuskal Wallis.

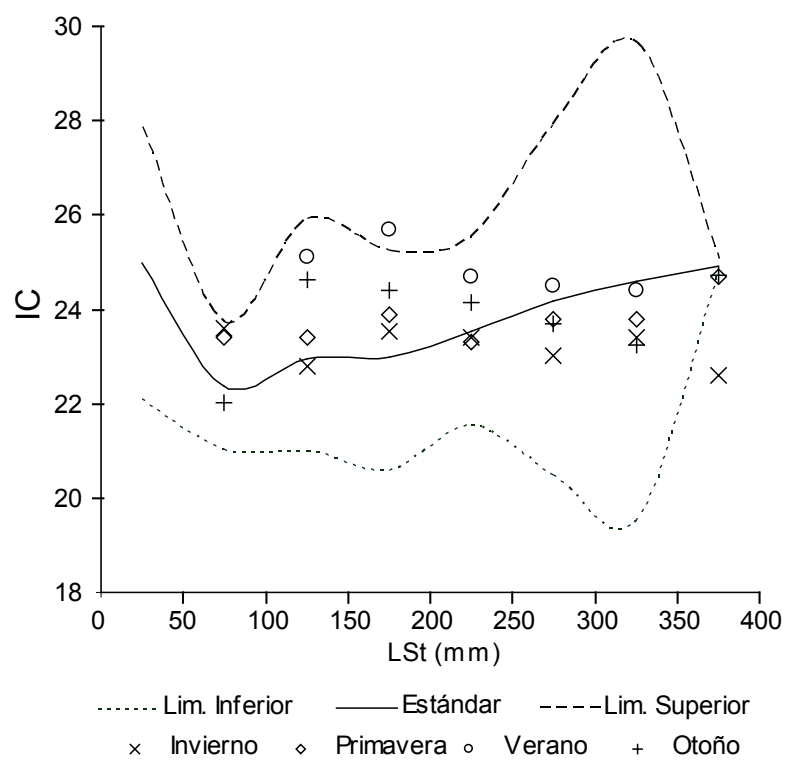

Figura 2. Índice cefálico (IC) de Odontesthes bonariensis de la laguna Los Charos.

\section{Resultados}

En los cuatro muestreos se capturaron 2862 ejemplares de un rango de talla comprendido entre 38 y $380 \mathrm{~mm}$ de LSt. La CPUE presentó variaciones importantes a lo largo del año (Tabla 1). En términos de biomasa, la CPUE fue de $74,3( \pm 71,0) \mathrm{kg} / 20$ hs de tendido de red. Por muestreo, la mayor captura por tren de enmalle se registró en invierno $(214,9 \mathrm{~kg})$, similar situación se registró con la cantidad de peces capturados. Al comparar el período total de estudio, la CPUE (en biomasa y número de ejemplares) de otońo fue 89,19 y $57,9 \%$ menor a la registrada en invierno. $\mathrm{Al}$ analizar capturas con idénticos artes de pesca, la CPUE media anual fue claramente superior al promedio de otras 23 lagunas pampeanas $(P<0,01)$. En relación a la pesca recreativa, se estimó una captura de $142 \mathrm{~kg} / \mathrm{ha} /$ año.

Los índices de condición corporal, arrojaron resultados que se ubicaron por encima y debajo de los valores estándar. En relación al índice cefálico, se destacó la regular condición de los peces jóvenes y una mejor tendencia de la misma en los ejemplares mayores a $250 \mathrm{~mm}$ de LSt (Fig. 2). En cuanto a K, los valores en general se ubicaron por debajo del estándar de la especie, a excepción de los registros de invierno en ejemplares mayores de $250 \mathrm{~mm}$ de LSt (Fig. 3). Por el contrario, los valores mas desfavorables de $\mathrm{K}$ se observaron en verano y otońo. Por su parte, el peso relativo presentó diferencias significativas entre muestreos

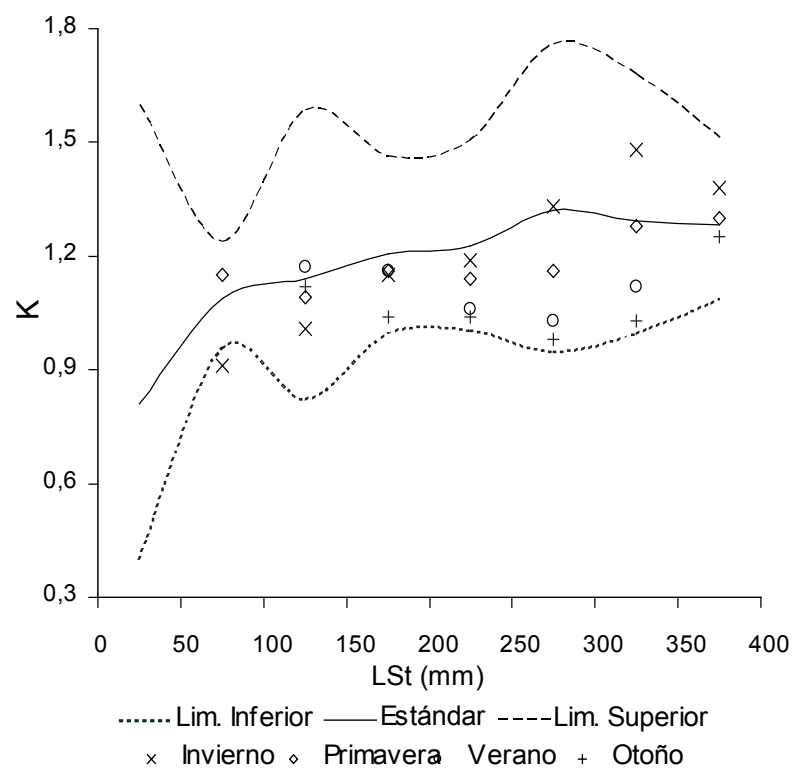

Figura 3. Índice de condición $(\mathrm{K})$ de Odontesthes bonariensis de la laguna Los Charos. 


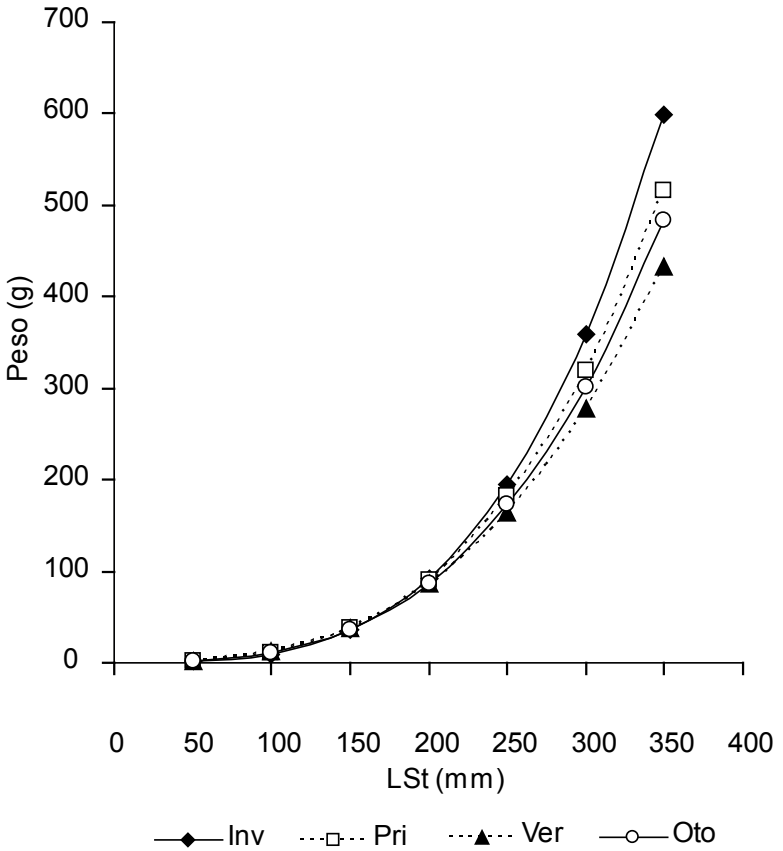

Figura 4. Relación LSt-peso de Odontesthes bonariensis de la laguna Los Charos.

$(P<0,01)$, con valores más altos también en invierno (Tabla 1$)$, en concordancia con un alto porcentaje de hembras en grado 4 y 5 de madurez gonadal (inicio de periodo de freza).

La relación LSt-peso también presentó diferencias significativas entre épocas del año $(P<0,01)$, en verano el valor del exponente b fue el más bajo (Fig. 4), mientras que la mejor relación se registró en invierno (Tabla 1). En la Figura 5 se puede observar la relación LSt-Longitud total, ésta fue:

$\mathrm{LT}(\mathrm{mm})=8,23+\mathrm{LSt}^{*} 1,14\left(\mathrm{n}=283 ; \mathrm{R}^{2}=0,99\right)$.

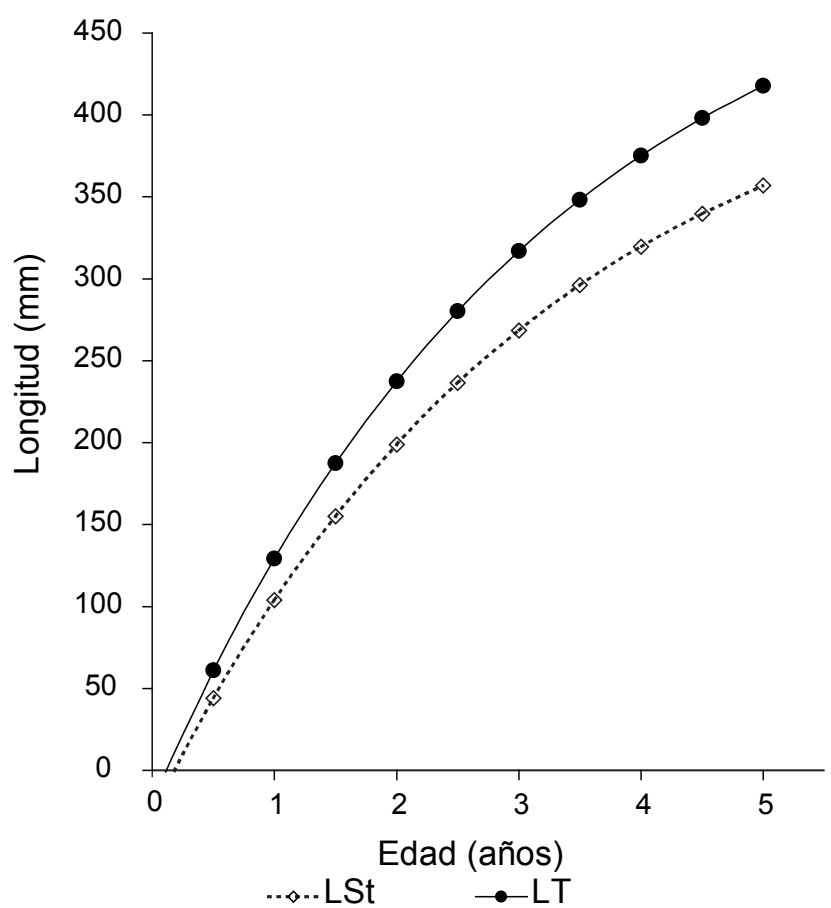

Figura 6. Crecimiento de Odontesthes bonariensis en la laguna Los Charos.

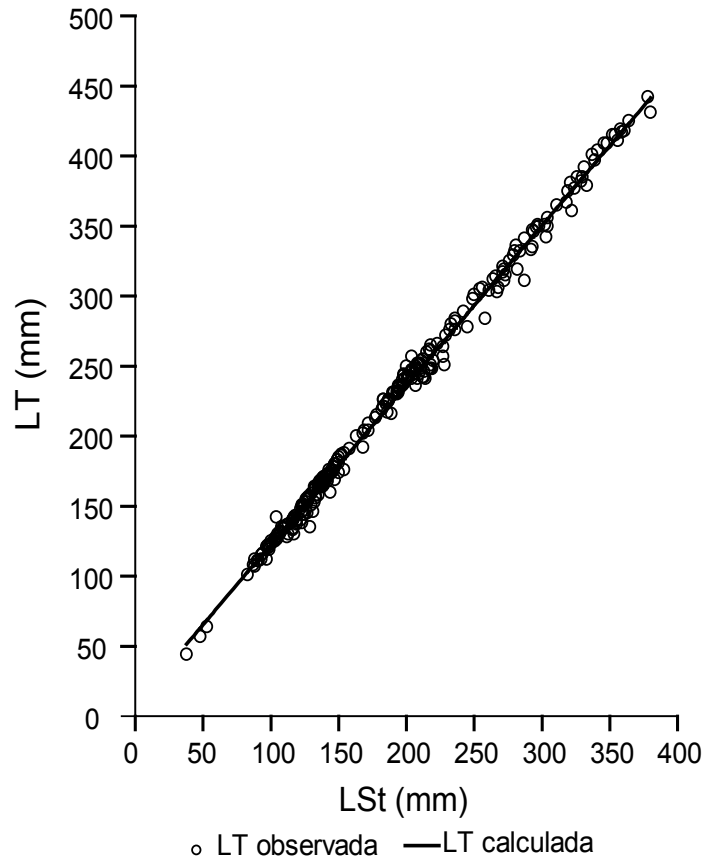

Figura 5. Relación LSt-LT de Odontesthes bonariensis en la laguna Los Charos.

El crecimiento calculado fue:

$$
\mathrm{LSt}_{t}=459,8^{*}\left[1-\exp ^{\left(-0,3105^{*}(\mathrm{t}-0,175)\right)}\right] \text { (Fig. 6). }
$$

El ejemplar de mayor tamaño fue capturado en verano con un registro de $442 \mathrm{~mm}$ de LT y 746,6 g de peso húmedo. El valor del índice de performance de crecimiento fue $\mathrm{W}=142,7$.

Con respecto a la ecología alimentaria, se observó una alimentación muy heterogénea. En ejemplares de hasta $120 \mathrm{~mm}$ de LSt, los copépodos, larvas de insectos y ostrácodos, fueron los alimentos más importantes (Tabla 2). En peces de 120 a $250 \mathrm{~mm}$ de LSt, el patrón fue similar, pero se observó además un importante consumo de caracoles y larvas de insectos. En estos dos grupos de peces, Copepoda estuvo presente prácticamente en todos las épocas, pero en ningún caso constituyó un ítem primario. Dicha situación coincide con la abundancia del macrozooplancton de la laguna, el cual presentó diferencias significativas entre épocas del año $(P<0,05)$, la mayor densidad se obtuvo en verano (Tabla 2). En pejerreyes de más de $250 \mathrm{~mm}$ de LSt, los ítems más importantes fueron caracoles y peces de la misma especie (canibalismo). Del análisis de todos los estadios de peces, Gastropoda representó un importante volumen del alimento ingerido.

En relación a la parasitosis, se detectó la presencia de Contracaecum sp. (Anisakidae), siendo frecuente en peces de $2+$ años de edad. La mayor prevalencia se registró en verano (24,6\%), la intensidad promedio fue más elevada en otoño (Tabla 3)

\section{Discusión}

La CPUE de $O$. bonariensis al igual que la distribución de tallas, exhiben amplias variaciones espaciales y temporales en las diferentes poblaciones que habitan las lagunas pampeanas (Baigún y Anderson, 1993). La mayor CPUE en biomasa y número de ejemplares capturados en invierno, se relaciona con la mayor probabilidad de captura y el mayor peso individual de los peces debido a la coincidencia con el inicio del período reproductivo. Esta captura estacional se considera muy elevada 
Tabla 2. Items alimenticios de Odontesthes bonariensis y abundancia de zooplancton (org/100 L de agua) en la laguna Los Charos.

\begin{tabular}{|c|c|c|c|c|c|c|c|c|c|c|c|c|}
\hline \multirow{2}{*}{$\begin{array}{l}\text { Epoca } \\
\text { Item }\end{array}$} & \multicolumn{3}{|c|}{ Invierno } & \multicolumn{3}{|c|}{ Primavera } & \multicolumn{3}{|c|}{ Verano } & \multicolumn{3}{|c|}{ Otoño } \\
\hline & I & II & III & I & II & III & I & II & III & I & II & III \\
\hline Gastropoda & $\mathrm{T}$ & $S$ & $\mathrm{P}$ & $\mathrm{A}$ & $S$ & $\mathrm{P}$ & A & $\mathrm{T}$ & $\mathrm{P}$ & $\mathrm{A}$ & $S$ & $\mathrm{P}$ \\
\hline Cladocera & $\mathrm{O}$ & $\mathrm{O}$ & $\mathrm{A}$ & $\mathrm{A}$ & A & A & A & $\mathrm{O}$ & A & $\mathrm{O}$ & A & $\mathrm{O}$ \\
\hline Chlorophyceae & $\mathrm{T}$ & $\mathrm{O}$ & $\mathrm{A}$ & $\mathrm{O}$ & $\mathrm{T}$ & A & $\mathrm{T}$ & $\mathrm{T}$ & A & $\mathrm{T}$ & $\mathrm{T}$ & A \\
\hline Copepoda & $\mathrm{T}$ & $\mathrm{T}$ & $\mathrm{A}$ & $\mathrm{T}$ & S & $\mathrm{T}$ & $S$ & $\mathrm{~T}$ & $\mathrm{~T}$ & S & S & A \\
\hline Cyanophyceae & A & A & $\mathrm{A}$ & $\mathrm{T}$ & $\mathrm{O}$ & A & $\mathrm{T}$ & $\mathrm{T}$ & A & $\mathrm{T}$ & S & A \\
\hline Bacillariophyceae & $\mathrm{T}$ & $\mathrm{T}$ & $\mathrm{A}$ & $\mathrm{T}$ & $\mathrm{T}$ & A & $\mathrm{O}$ & $\mathrm{O}$ & A & A & A & A \\
\hline Insecta & $\mathrm{T}$ & S & A & S & $\mathrm{T}$ & A & $S$ & S & A & S & $\mathrm{T}$ & A \\
\hline Ostracoda & $\mathrm{T}$ & $\mathrm{O}$ & $\mathrm{A}$ & S & $\mathrm{T}$ & A & S & S & A & S & S & A \\
\hline Peces & A & $\mathrm{O}$ & $\mathrm{P}$ & $\mathrm{A}$ & A & $\mathrm{P}$ & A & A & $\mathrm{P}$ & A & A & $\mathrm{P}$ \\
\hline Restos vegetales & A & $\mathrm{T}$ & $\mathrm{O}$ & A & A & $\mathrm{T}$ & A & A & $\mathrm{O}$ & $\mathrm{A}$ & A & A \\
\hline Rotifera & $\mathrm{T}$ & $\mathrm{T}$ & $\mathrm{A}$ & $\mathrm{T}$ & $\mathrm{T}$ & A & $\mathrm{T}$ & $\mathrm{T}$ & A & $\mathrm{T}$ & A & A \\
\hline Items no clasificados & $\mathrm{T}$ & $\mathrm{T}$ & $\mathrm{A}$ & A & $\mathrm{O}$ & $\mathrm{A}$ & A & $\mathrm{O}$ & $\mathrm{A}$ & $\mathrm{A}$ & $\mathrm{O}$ & $\mathrm{A}$ \\
\hline Densidad zooplancton & \multicolumn{3}{|c|}{$1625 \pm 926$} & \multicolumn{3}{|c|}{$9162 \pm 2635$} & \multicolumn{3}{|c|}{$13187 \pm 3715$} & \multicolumn{3}{|c|}{$4062 \pm 1214$} \\
\hline Rel. Copepoda-Clacodera & \multicolumn{3}{|c|}{ 19:1 } & \multicolumn{3}{|c|}{$31: 1$} & \multicolumn{3}{|c|}{ 105:1 } & \multicolumn{3}{|c|}{$325: 1$} \\
\hline
\end{tabular}

I: Peces > 120 mm de LSt. II: Peces de 120 - 250 mm de LSt. III: Peces > 250 mm de LSt. P: alimento primario. S: alimento secundario. T: alimento terciario. O: alimento ocasional. A: ausente.

y confirma la importante producción de pejerrey que pueden alcanzar ciertas lagunas pampeanas. Por su parte, el valor medio anual de abundancia es superior a valores de referencia de la región centro-este de Argentina (Grosman et al. 2001; 2005; Mancini y Grosman 2001; 2004), y sería la respuesta a la falta de explotación en los primeros años posteriores a la siembra de alevinos. La disminución consecutiva de CPUE se le atribuye a una mortandad registrada en verano y a la fuerte presión de pesca recreativa (menor peso individual de los ejemplares), la cual fue mayor a valores medios de referencia estimados en otras lagunas pampeanas (Mancini y Grosman 2004).

Una baja condición corporal de los peces coincidió con la menor CPUE, por lo que no podría adjudicarse dicha situación a fenómenos de densodependencia. Del análisis conjunto de los índices de condición y de la relación longitud-peso, se desprende que de acuerdo a la época del año, la condición mas desfavorable se presentó en verano, donde existió la mayor densidad de zooplancton. De acuerdo al estudio realizado por Mancini et al. (2004), la presencia de Contracaecum sp. en ejemplares de $2+$ años de vida, es uno de los factores más importantes que se asoció con la disminución de la condición corporal. Por el contrario y de manera conjunta con una menor carga parasitaria y el inicio de la actividad reproductiva de $O$. bonariensis, en invierno se registró la mejor condición.

Diferentes factores climáticos, ambientales y poblacionales intervienen en la velocidad de crecimiento de una especie íctica, en donde la densidad poblacional suele ser uno de los más importantes (Guerra Sierra y Sánchez Lizaso 1998). A pesar de la gran abundancia de pejerrey que presenta la laguna Los Charos, los valores de crecimiento se encuentran dentro del rango de referencia de la especie, aunque relativamente bajo hasta el año de

Tabla 3. Prevalencia, abundancia e intensidad promedio de Contracaecum sp. en Odontesthes bonariensis de la laguna Los Charos.

\begin{tabular}{lcccc}
\hline Epoca & Invierno & Primavera & Verano & Otoño \\
\hline Prevalencia & 5,3 & 12,0 & 24,6 & 4,1 \\
Abundancia & 0,06 & 0,44 & 0,94 & 0,23 \\
Intensidad media & 1,3 & 3,7 & 3,8 & 5,6 \\
\hline
\end{tabular}

vida. La ausencia de competencia interespecífica en función de la baja riqueza que presenta el ambiente bajo estudio (3 especies), donde $O$. bonariensis representa más del $97 \%$ de las capturas (Nicola et al. 2007) y las diferencias de las marcaciones anuales de las distintas cohortes por el tipo particular de reproducción que posee el pejerrey (Sendra y Colautti 1997), podrían contribuir a explicar dicha situación.

La naturaleza del alimento ingerido por los peces depende principalmente de la morfología y del comportamiento alimentario de la especie y en segundo lugar, de la composición y cantidad de alimento disponible (Zavala-Camin 1996). El hábitat trófico del pejerrey es en general pelágico, los ejemplares menores a los dos años de vida consumen preferentemente zooplancton, ocupando el tercer nivel trófico. Sin embargo, cuando el zooplancton es escaso, el pejerrey presenta una versatilidad anatómica que le permite ampliar su espectro trófico (Ringuelet et al. 1980). En concordancia con ello, el pejerrey de la laguna Los Charos presenta una alimentación heterogénea, producto seguramente de las oscilaciones en la oferta de zooplancton. En ejemplares de hasta 2 ańos de vida, Copepoda fue un componente importante de la dieta pero en ningún momento los componentes del zooplancton representaron un alimento primario a diferencia de una amplia variedad de poblaciones evaluadas (Burbidge et al. 1974; Bahamondes et al. 1979; Grosman 1995; Mancini y Grosman 1998; Escalante 2001; Silva Cassemiro et al. 2003) y explicaría el bajo crecimiento inicial de los peces. El mayor consumo de organismos bentónicos como caracoles y larvas de insectos, estaría en parte cubriendo el déficit de zooplancton, además de la modificación de la dieta conforme a los grupos de tamaño de los peces (Bemvenuti 1990; Piedras y Pouey 2005).

En ejemplares mayores a $250 \mathrm{~mm}$ de LSt, los ítems más importantes coinciden con los reportes de otros autores (Burbidge et al. 1974; Escalante 2001; Mancini y Grosman 2004). La gran disponibilidad de pejerreyes juveniles explicaría la mejor condición corporal observada en dicha talla durante el invierno y marca un cambio en la condición de $O$. bonariensis cuando modifica su dieta de zooplanctívoro a piscívoro, aunque es importante destacar el sesgo propio del desove y las cargas parasitarias mencionadas. 
En conclusión, la laguna Los Charos exhibe una elevada producción inicial de $O$. bonariensis. El grado de desarrollo gonadal, el cambio de la dieta y la presencia de Contracaecum sp., interfieren sobre la condición corporal de los peces. El crecimiento del pejerrey es icicialmente bajo pero luego se encuadra dentro de valores de referencia. A diferencia de una gran variedad de poblaciones evaluadas, el zooplancton no constituye un ítem alimenticio primario del pejerrey. La alternancia de periodos de sequía e inundación, tendrá un rol clave en el futuro aprovechamiento de la laguna.

\section{Agradecimientos}

A los propietarios de la laguna Los Charos. A Manuel Ortiz y Armando Nilson por la colaboración brindada en las tareas de campo. A la SECyT de la Universidad Nacional de Río Cuarto, Argentina.

\section{Literatura citada}

Bahamondes, I., D. Soto \& I. Vila. 1979. Hábitos alimentarios de los pejerreyes (Pisces, Atherinidae) del lago Rapel, Chile. Medio Ambiente 4(1): 3-18.

Baigún, C. \& R. Anderson. 1993. Structural indices for stock assessment of and management recomendations for pejerrey Odontesthes bonariensis in Argentina. North American J. of Fisheries Management 13: 600-608.

Baigún, C. \& R. Delfino. 2001. Consideraciones y criterios para la evaluación y manejo de pesquerías de pejerrey en lagunas pampásicas. In: Fundamentos biológicos, económicos y sociales para una correcta gestión del recurso pejerrey. F. Grosman (Ed.). Ed. Astyanax, Azul, Buenos Aires. Pp. 132-145.

Bemvenuti, M. 1990. Hábitos alimentares de peixe-rei (Atherinidae) na regiao estuarina da lagoa Dos Patos, RS, Brasil. Atlantica, Rio Grande 12(1): 79-102.

Bemvenuti, M. 2006. Silversides in South Brazil: Morphological and ecological aspects. Biocell 30(1): 111-118.

Burbidge, R., M. Carrasco \& P. Brown. 1974. Age, growth, lengthweight relationship, sex ratio and food habitats of the Argentine pejejerrey, Basilichthys bonariensis (Cuv. and Val.), from lake Peñuelas, Valparaíso, Chile. J. Fish. Biol. 6:299-305.

Calvo, J. \& L. Dadone. 1973. Fenómenos reproductivos en el pejerrey (Basilichthys bonariensis). I: Escala y tabla de madurez. Rev. Museo de La Plata, Zool, XI(102):123-163.

Cantero, A., M. Cantú, J. Cisneros, J. Cantero, M. Blarasin, A. Degioanni, J. Gonzalez, V. Becerra, H. Gil, J. de Prada, S. Degiovanni, C. Cholaki, M. Villegas, A. Cabrera \& C. Eric. 1998. Las tierras y aguas del sur de Córdoba. Propuestas para un manejo sustentable. Universidad Nacional de Río Cuarto, Argentina.

Colautti, D., M. Remes Lenicov \& G. Berasain. 2003. Vulnerabilidad del pejerrey Odontesthes bonariensis a la pesca deportiva en función de su condición. Biología Acuática 20: 49-55.

Dangvas, N. 1998. Los ambientes lénticos de la pampasia bonaerense, República Argentina. In: Cirelli, A. (Comp.). Agua. Problemática regional. Editorial Universitaria Buenos Aires. Buenos Aires. Pp. 145-149.

Escalante, A. 2001. Alimentación natural del pejerrey. In: Fundamentos biológicos, económicos y sociales para una correcta gestión del recurso pejerrey. F. Grosman (Ed.). Ed. Astyanax, Azul, Buenos Aires. Pp. 67-75.

Fabian, D. 1999. Zooplancton. In: R. Arocena y D. Conde (Eds.). Métodos en ecología de aguas continentales. Montevideo, Uruguay. Pp. 165-181.
Freyre, L. 1976. Normas para la inspección y determinación de ambientes pesqueros pampásicos. La Plata: Dir. Rec. Nat. Min. Asuntos Agrarios. Argentina.

Freyre, L., L. Protogino \& J. Iwaskiw. 1983. Demografía del pejerrey Basilichthys bonariensis bonariensis en el embalse Río Tercero, Córdoba. Descripción de las artes de pesca. Biología Acuática 4: $39 \mathrm{p}$.

Gallucci, V. \& T. Quinn. 1979 Reparameterizing, fitting, and testing a simple growth model. Trans. Am. Fish. Soc. 108:14-25.

García de Jalón Lastra, D, M. Mayo Rustarazo, F. Hervella Rodriguez, E. Barceló Culebras \& T. Fernández Couto. 1993. Principios y técnicas de gestión de la pesca en aguas continentales. Ed. Mundi-Prensa, Madrid.

García-Berthou, E. 2001. On the missue of residuals in ecology: testing regression residuals vs. the analysis of covariance. Journal of Animal Ecology 70: 708-711.

Grosman, F. 1995. Variación estacional de la dieta del pejerrey (Odontesthes bonariensis). Rev. Asoc. Cs. Nat. Litoral 26 (1): 9-18.

Grosman, F. \& M. Mancini. 2001. Alcances socioeconómicos de la pesca deportiva del pejerrey. Realidad Económica 184: 106-121.

Grosman F, P. Sanzano, D. Agueria \& G. Gonzalez. 2001. Gestión del pejerrey Odontesthes bonariensis en una pesquería periurbana de Argentina. AquaTIC 14: 1-15.

Grosman F, P Sanzano \& G Rudzik. 2005. Diagnóstico limnológico pesquero de 6 lagunas del partido de Puán. Propuesta de pautas de gestión del recurso. Biología Acuática 22: 177-188.

Guerra Sierra, A. \& J. Sánchez Lizaso. 1998. Fundamentos de explotación de recursos vivos marinos. Zaragoza: Editorial Acribia.

López, H., C. Baigún, J. Iwaszkiw, R. Delfino \& O. Padin. 2001. La cuenca del Salado: uso y posibilidades de sus recursos pesqueros. La Plata: Ed. de la Universidad de La Plata.

Mancini, M. \& F. Grosman. 1998. Aspectos poblacionales del pejerrey (Odontesthes bonariensis) en el embalse Río Tercero (Córdoba), Argentina. Natura Neotropicalis 29(2): 137-143

Mancini, M. \& F. Grosman. 2001. Efecto de la pesca deportiva sobre una población de pejerrey (Odontesthes bonariensis). In: Grosman, F. (Ed.). Fundamentos biológicos, económicos y sociales para una correcta gestión del recurso pejerrey. Ed. Astyanax, Azul. Pp. 105-110.

Mancini, M. \& F. Grosman. 2004. Estructura y funcionamiento de la pesquería recreacional del pejerrey Odontesthes bonariensis en la laguna de Suco, Córdoba, Argentina. AquaTIC 20: 20-31.

Mancini, M., I. Nicola, A. Larriestra \& V. Salinas. 2004. Patrones de riesgo e implicancias de la presencia de Contracaecum sp. (Nematoda, Anisakidae) en pejerrey Odontesthes bonariensis (Pisces, Atherinopsidae). Biología Acuática 22: 197-202.

Margolis, L., G. Esch, J. Colmes, A. Kuris \& G. Schad. 1982. The use of ecological terms in parasitology. J. Parasitology 68(1): 131-133.

Moreau, J. 1987. Mathematical and biological expresión of growth in fishes: recent trends and further developments. In: R. Summerfelt \& G. Hall (Eds). Age and Growthof Fish. Iowa State University Press. Unites States of America. Pp. 81-113.

Nicola, I., M. Mancini, V. Salinas, C. Bucco \& C. Rodriguez. 2007. Caracterización de humedales. La laguna pampeana Los Charos (Córdoba, Argentina). Gestión Ambiental 13(1): 21-32. 
Piedras, S. \& J. Pouey. 2005. Alimentacao do peixe-rei (Odontesthes bonariensis, Atherinopsidae) nas lagoas Mirim e Mangueira, Rio Grande do Sul, Brasil. Iheringia, Sér. Zool. 95(2): 117-120.

Quirós, R., J. Rosso, A. Renella, A. Sosnovsky \& M. Boveri. 2002. Análisis del estado trófico de las lagunas pampeanas (Argentina). Interciencia 27(11): 584-591.

Quirós, R. 2004. Sobre la morfología de las lagunas pampeanas. Serie de Documentos de Trabajo del Area de Sistemas de Producción Acuática. Fac. de Agronomía, Universidad de Buenos Aires. $15 \mathrm{p}$.

Ringuelet, R., R. Iriart \& A. Escalante. 1980. Alimentación del pejerrey (Basilichthys bonariensis, Atherinidae) en la laguna Chascomus (Buenos Aires, Argentina). Relaciones ecológicas de complementación y eficiencia trófica del plancton. Limnobios 1(10): 447-460.
Royce, W. 1996. Introduction to the Practice of Fishery Science. California: Academic Press.

Sendra, E. \& D. Colautti. 1997. Procedimiento metodológico para el estudio del crecimiento del pejerrey Odontesthes bonariensis bonariensis de la laguna de San Miguel del Monte, Prov. Buenos Aires, Argentina. Natura Neotropicalis 28(2):105-115.

Silva Cassemiro, F., N. Hahn \& T. de Rangel. 2003. Diet and trophic ecomorphology of the silverside, Odontesthes bonariensis, of the Salto Caxias reservoir, rio Iguacu, Paraná, Brazil. Neotropical Ichthyology 1(2): 127-131.

Weatherley, A. 1976. Growth and ecology of fish populations. London: Academis Press.

Zavala-Camin, L. 1996. Introducao aos estudios sobre alimentacao natural em peixes. Maringá (Brasil): EDUEM. 


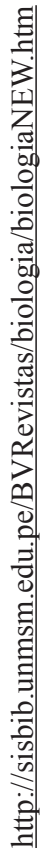

